\title{
Effects of fiber volume fraction on unidirectional kenaf/epoxy composites: the transition region
}

\begin{abstract}
This article presents the effects of fiber volume fraction on the existence of a transition region in unidirectional kenaf/epoxy composites. The composites were made from hand Lay-up techniques, with three formulations of fiber volume fraction employed: $0 \%$ (neat), $15 \%$ and $45 \%$. The results showed that tensile properties such as tensile strength and modulus of elasticity increased as the fiber volume increased. The stress-strain curves showed that the kenaf/epoxy composites exhibited bi-linear responses with reductions in the modulus of elasticity. Surface morphology aided by a scanning electron microscope (SEM) revealed that the reduction in the modulus of elasticity was due to matrix cracking.
\end{abstract}

Keyword: Composite; Epoxy resin; Kenaf fibre 